\title{
Evaluación de los catálogos en línea de las unidades de información especializadas en Costa Rica: una propuesta metodológica
}

\section{Zelenia Rodríguez Morales}

*Philosophiae Doctor (Ph.D.) en Bibliotecología de la Universidad Autónoma de Centro América. Jefa de la Unidad de Documentación del Instituto Nacional de las Mujeres, San José Costa Rica; zrodriguez@inamu.go.cr

Recibido: 15 de enero del 2013
Aceptado: 5 de marzo del 2013

\section{RESUMEN}

El artículo presenta los resultados de la investigación Evaluación de los catálogos en línea de las unidades de información especializadas en Costa Rica: una propuesta metodológica. El principal objetivo de esta investigación fue elaborar una propuesta de evaluación que permitiera conocer el estado del catálogo en línea, particularmente en lo relativo a la búsqueda, la web social y la interfaz gráfica, y verificar que se ajuste a los requerimientos actuales. La adaptación de una metodología de evaluación de catálogos en línea implicó el análisis de distintos sistemas evaluativos aplicados en unidades de información nacionales e internacionales de este importante servicio. La metodología adaptada se validó en los catálogos en línea del Instituto Nacional de las Mujeres (INAMU), el Instituto Interamericano de Derechos Humanos (IIDH) y la Corte Interamericana de Derechos Humanos (CIDH), todas con sede en Costa Rica, cuyas unidades de información han realizado acciones que tienden a garantizar a sus personas usuarias un catálogo en línea especializado en la temática de los derechos humanos.

\section{PALABRAS CLAVE}

Catálogos en línea, web social, evaluación de catálogos en línea, Biblioteca 2.0

\section{SUMMARY}

This paper presents the results of the research The assessment of the online catalogs of specialized information units in Costa Rica: a methodological proposal. The main objective of this research was to develop a proposal that would enable assessment of the catalogs online, particularly with respect to information search, social web and interface, and ensure that it conforms to the current requirements. The methodology of evaluation of online catalogs involved the analysis of different assessment systems applied to national and international units of information. The adapted methodology was validated in the online catalogs of the National Institute of Women (INAMU), American Institute of Human Rights (IIHR) and the Inter-American Court of Human Rights (IACHR), whose information units have taken actions that ensure their users an online catalog specializing mainly in the area of human rights.

\section{KEY WORDS}

Online public access catalog, social web, online public access catalog evaluations, library 2.0

\section{INTRODUCCIÓN}

Esta investigación titulada la Evaluación de los catálogos en línea de las unidades de información especializadas en Costa Rica se desarrolló con base en cuatro aspectos fundamentales. El primero de ellos fue el estudio de los avances de los catálogos en línea de acceso público, principalmente 
en las instituciones del sector público costarricense y en universidades públicas y privadas de Costa Rica.

Además del marco jurídico que regula los diferentes aspectos del acceso a la información pública, desde noviembre del 2004, la Defensoría de los Habitantes de la República impulsa la Red Interinstitucional de Transparencia, una iniciativa que tiene como objetivo promover la divulgación de información producida por las instituciones del Estado costarricense. Otro esfuerzo del Estado costarricense por democratizar el acceso y uso de la información principalmente del ámbito administrativo es el Gobierno Digital.

En este sentido, las nuevas tecnologías de información y comunicación son herramientas efectivas para incrementar la disponibilidad de la información que poseen las instituciones públicas. Uno de estos instrumentos eficaces para almacenar y difundir esta información son los catálogos en línea que poseen las unidades de información especializadas, medios públicos y de fácil acceso.

El segundo aspecto explorado en la evolución de los catálogos en línea es la llamada web social, que implica una participación ciudadana más activa y el compromiso de las personas por compartir recursos y generar nuevos conocimientos a partir de los mismos. La web social se construye a partir de un grupo de personas usuarias; en ella, el conocimiento de cada una, sumado al de otras, contribuye a formar y fortalecer lo que algunas autorías llaman el conocimiento colectivo.

En las unidades de información es el catálogo en línea donde mejor se demuestra este aprovechamiento de la inteligencia colectiva. Las personas usuarias, con sus comentarios, puntuaciones, etiquetas y reseñas crean conocimiento que se añade al catálogo bibliográfico y de esta forma se enriquece colectivamente.

Así, el tercer factor que involucra esta investigación se refiere a la evaluación como medio de avance y mejora constante que permite a las unidades de información perfeccionar la propuesta que presenta el catálogo en línea. La evaluación ha sido una herramienta utilizada en diferentes espacios sociales para dar cuenta del cumplimiento de los objetivos y las metas propuestas, y los catálogos en línea no son la excepción.

Se considera importante realizar estudios de evaluación de los catálogos en línea, con el propósito de conocer si cumplen con los objetivos para los cuales fueron implementados. Dentro de los parámetros que se podrían evaluar, está la interfaz gráfica, con el fin de determinar si es amigable. Otro parámetro es el relacionado con la satisfacción de los requisitos de usabilidad y accesibilidad. Además este tipo de evaluaciones permite verificar el rendimiento de los recursos presupuestados, la forma en que las tareas se están llevando a cabo; además, observar el desarrollo del servicio y planificar en un contexto de cambio, a fin de ofrecer a las personas usuarias un servicio que responda ampliamente a sus necesidades.

Por último, una exploración previa realizada para efectos de la investigación evidenció que las prácticas evaluativas de catálogos en línea en Costa Rica son escasas y, por lo tanto, sus proyecciones de desarrollo se ven limitadas en relación con los procesos actuales de cambio.

Así, esta investigación pretende convertirse en un instrumento que permita a las unidades de información especializadas, como la del Instituto Nacional de las Mujeres (INAMU), el Instituto Interamericano de Derechos Humanos (IIDH) y la Corte Interamericana de Derechos Humanos (CIDH), evaluar sus catálogos en línea de acuerdo con los avances tecnológicos de los últimos tiempos, el incremento de las redes sociales y de comunicación, y las demandas de información de personas usuarias con características sociales, culturales y económicas cada vez más diversas y exigentes. 


\section{Marco teórico}

El Diccionario Enciclopédico de Ciencias de la Documentación (2004, p. 273) define catálogo como una palabra procedente del latín catalogus, que a su vez proviene del vocablo griego catálogo, el cual se forma de dos raíces: kata (con orden) + lego (yo digo).

Para efectos de la investigación, se asumió la siguiente definición del catálogo en línea, la cual se acuña con base en diferentes acepciones estudiadas como un "instrumento que permite a las personas usuarias acceder y consultar de forma intuitiva, interactiva y amigable los materiales que componen una colección real o virtual a través de una computadora".

Las tres generaciones de catálogos en línea definidas por el profesor Hildreth, en 1984, han servido para marcar la evolución histórica de los mismos y comprender el proceso que han seguido. A esta distinción, Fattahi (1997), en su tesis doctoral, propone una cuarta generación marcada por las interfaces gráficas de personas usuarias, y en los últimos años se ha escrito sobre la última generación de catálogos en línea, donde la interfaz gráfica es el propio navegador de internet.

Por otro lado, y dada la importancia de la web social en el estudio, se define como una herramienta que facilita el aprovechamiento de la inteligencia colectiva, ya que permite aportar, etiquetar, opinar, valorar y utilizar conocimientos presentados por otras personas. Es decir, las personas usuarias colaboran con las unidades de información en la producción de nuevos contenidos para el catálogo en línea.

Algunas autorías, como, Margaix-Arnal (2007, 2008), Marcos Mora (2009) y Sans Paricio (2010) consideran herramientas y servicios de la web 2.0, las redes sociales, la sindicación de contenidos, los marcadores sociales, wikis, blogs, podcasting, entre otros.

Por otro lado, a partir de la web social se abordaron dos conceptos utilizados recientemente en las unidades de información, como son: la biblioteca 2.0 y el catálogo en línea 2.0.

La Biblioteca 2.0 es la aplicación de los servicios y herramientas de la web social de tipo interactivo, colaborativo y multimedia, tales como: videos, fotografías, chats, redes sociales, entre otros.

Por su parte, el catálogo en línea 2.0 aplicación de las tecnologías y las actitudes de la Web social al catálogo bibliográfico. Por ejemplo, elaboración de reseñas, establecer puntuaciones, realizar etiquetas y comentarios.

En la Figura 1 se muestra el concepto de la web social en relación con sus herramientas y servicios.

Finalmente, para esta investigación, la evaluación es una herramienta que permite conocer mejor y más a fondo los sistemas de información, elegir entre diferentes opciones, realizar actividades de mejora cuando sea necesario, o suprimir servicios cuando ya no son de utilidad para las personas usuarias. Por lo tanto, la evaluación consiste en emitir un juicio de valor o una apreciación de las ventajas de un servicio.

Así, la evaluación es la puesta en práctica de un procedimiento con el cual se obtienen las cualidades, ventajas y debilidades del servicio de información que se está valorando; de esta forma, es posible mejorar el servicio actual, de tal forma que se ajuste a las necesidades de las personas usuarias.

\section{METODOLOGÍA}

La investigación se dividió principalmente en dos momentos: en el primero se realizó un análisis e interpretación de documentos relacionados con metodologías de evaluación de catálogos en línea, para lo cual se utilizaron técnicas y herramientas de la hermenéutica, como disciplina fundamental utilizada en los procesos de investigación de las ciencias sociales. 


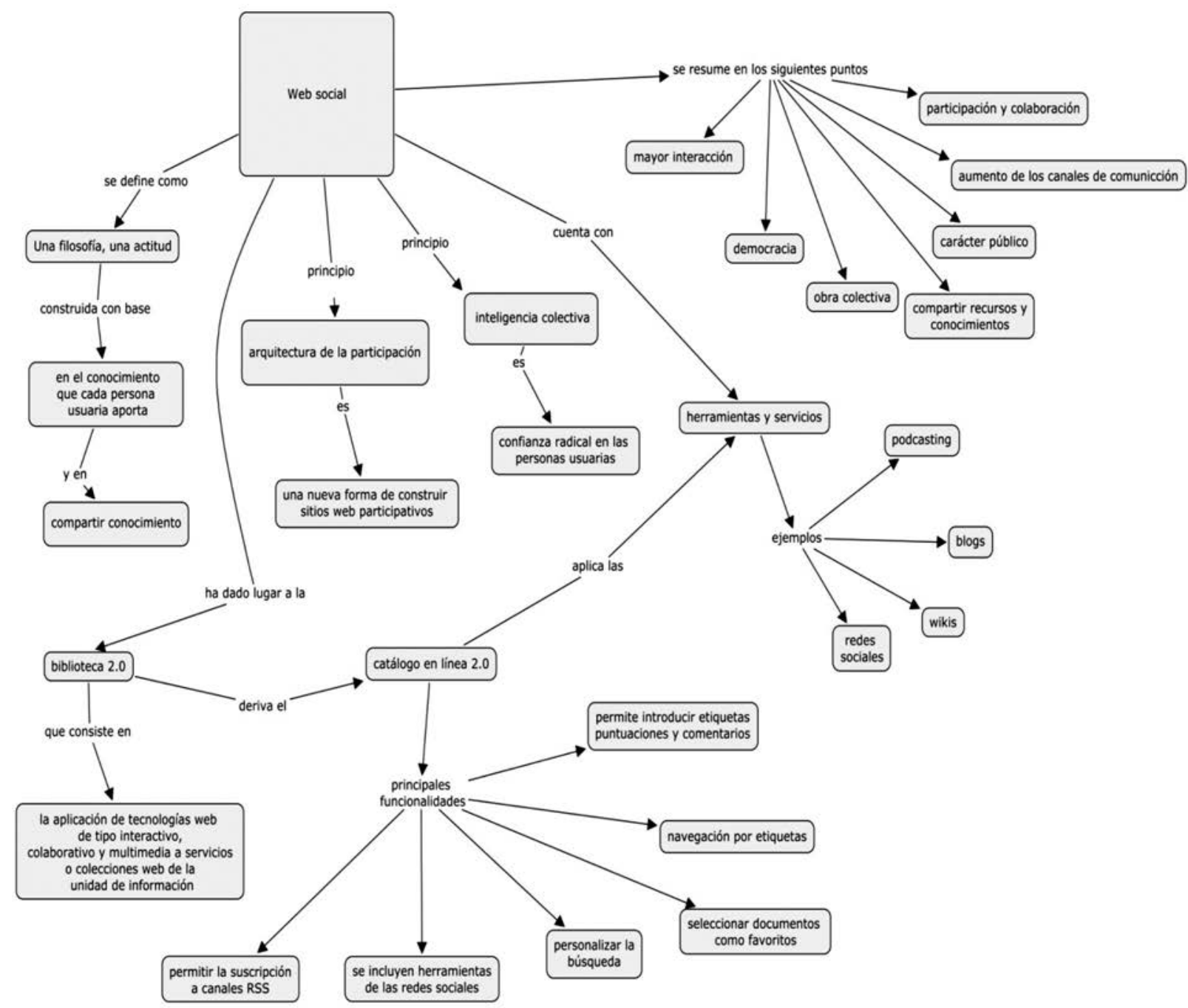

Figura 1. Web social.

Elaboración propia con base en las autorías estudiadas en la investigación, 2011.

En un segundo momento, se aplicaron herramientas del paradigma cuantitativo, principalmente en lo relativo a la obtención de evidencias de validez para el instrumento de evaluación, aplicado a tres catálogos en línea de unidades de información especializadas de las instituciones definidas.

En el proceso metodológico de diseño y adaptación de una metodología de evaluación de los catálogos en línea de las unidades de información especializadas, se llevaron a cabo las siguientes fases:

\section{Primera fase. Análisis de los diferentes estudios de evaluación}

Se identificaron las metodologías por revisar en la bibliografía y se realizó su interpretación y análisis, a fin de elegir la metodología que mejor se adaptara a los objetivos de la investigación. Fueron identificadas quince metodologías de 
evaluación. Estos estudios fueron ejecutados en diversos países, entre otros, Canadá, España, Argentina, Paraguay y Perú.

Este análisis tomó en consideración aspectos tales como: tipo de unidades de información donde se aplicó, las personas autoras en las cuales se basaron quienes realizaron la evaluación, los parámetros evaluados y las principales recomendaciones resultantes de la aplicación de esas propuestas metodológicas.

Se profundizó en el conocimiento de los estudios de autorías reconocidas en el ámbito bibliotecológico, como lo son: Juliana Chan, J. M. Cherry, C. Travieso Rodríguez, Purificación Moscoso Castro, Luisa Alvite Diez.

\section{Segunda fase. Encuesta de opinión a personas usuarias de los catálogos en línea}

Se conoció la opinión de las personas acerca del servicio del catálogo en línea que se utiliza en la unidad de información especializada donde se validó la propuesta metodológica.

\section{Tercera fase. Adaptación de la propuesta}

La adaptación de la propuesta se realiza con base en diversas metodologías y documentos analizados.

Principalmente, la metodología se desarrolla a partir de los principios de diseño de interfaces publicados en el 2005 por la IFLA, con el título Guidelines for online public access catalogue (OPAC) displays. Esta guía recopila un compendio de buenas prácticas en forma de directrices que ayudan a las unidades de información, especialmente las de carácter general, a diseñar o rediseñar la presentación del catálogo en línea, e indudablemente contribuyen a esta propuesta metodológica en la formulación de indicadores asociados a la visualización de los registros de autoridad, la navegabilidad del catálogo y en la aplicación de los formatos de visualización de las personas con necesidades especiales, entre otros.
Para efectos de la investigación, la plataforma para formular el instrumento para la evaluación del catálogo en línea en entorno web fue la metodología desarrollada en el año 2004, por Rodríguez Bravo y Alvite Díez, llamada Propuesta metodológica de evaluación de interfaces de OPACs. INNOPAC versus UNICORN, la cual fue aplicada en diez bibliotecas universitarias, y dada su reproducibilidad, también fue empleada en tres bibliotecas de museos españoles.

Por otro lado, este instrumento se complementa a la luz de las siguientes metodologías:

»S. Texidor y I. Muzylo. Form5o: un instrumento para análisis comparativo de OPACs. Aporta al instrumento de evaluación por su característica principal, ya que fue desarrollado para un curso de Procesamiento y Tecnologías de la Información del programa de la carrera de Bibliotecología y Documentación de la Universidad del Museo Social Argentino, en la ciudad de Buenos Aires.

»C. Travieso Rodríguez, J. A. Arévalo y J. M. Vivancos Secilla. Usabilidad de los catálogos de las bibliotecas universitarias: propuesta metodológica de evaluación. Así, este trabajo constituye una importante fuente de información que aporta una aplicación de los conceptos de la usabilidad y accesibilidad en catálogos de bibliotecas universitarias.

Con respecto a los indicadores de la web social D. Margaix Arnal (2008) desarrolla en diversos artículos la aplicación de los servicios y herramientas en el catálogo bibliográfico, entre ellos, EI OPAC 2.0: puerta de acceso a los contenidos de la biblioteca presentado en el IV Congreso Nacional de Bibliotecas Públicas del Ministerio de Cultura de Madrid, España.

Así, en la adaptación del sub parámetro Sistema de ayuda a personas usuarias, se tomaron como guía los artículos de Francisco GarcíaOrtiz y Purificación Moscoso Castro, titulados 
El sistema de ayuda al usuario de un catálogo en línea de acceso público: delimitación teórica y propuesta práctica (2007) y Análisis comparado de los sistemas de ayuda en opacs: tipología y tendencias (2007).

Finalmente, se consideró el estudio de Abadal Falgueras, E. (2002 setiembre - octubre). Elementos para la evaluación de interfaces de consulta de bases de datos web.

\section{- Parámetros y sub parámetros del instrumento de evaluación}

La metodología diseñada consta de tres parámetros fundamentales: las prestaciones de búsqueda, compuesta por 42 indicadores; la web social, que consta de 15 indicadores, y la interfaz gráfica, que consta de 31 indicadores. A su vez, cada uno se divide en sub parámetros.

Las prestaciones de búsqueda constan de los siguientes sub parámetros:

1. Página inicial del catálogo: los indicadores de este apartado constan de ocho indicadores.

2. Llaves del sistema de búsqueda: este sub parámetro está integrado por quince indicadores.

3. Página de resultados: está compuesta por catorce indicadores.

4. Gestión de contenidos: se compone de cinco indicadores en la primera sesión de valoración.

El parámetro dedicado a la web social lo componen quince indicadores y ningún sub parámetro.

Las características de la interfaz gráfica se dividieron en los siguientes sub parámetros:

1. Disposición visual: compuesto por diez indicadores.

2. Ergonomía: consta de ocho indicadores.

\section{Sistema de ayuda a las personas usua-}

rias: formado por trece indicadores.

\section{Cuarta fase. Validez de contenido}

Para la obtención de evidencias de validez del contenido de los indicadores, así como para la precisión y delimitación del alcance respectivo se realizaron dos acciones concretas: en primer lugar, se contó con la colaboración del juicio de personas expertas en bibliotecología e informática, y en segundo lugar, se realizó una prueba piloto que consistió en la aplicación del instrumento en una unidad de información seleccionada.

\section{Quinta fase. Relevancia del instrumento}

La relevancia del instrumento se llevó a cabo con la colaboración de tres profesionales en bibliotecología seleccionadas del grupo de personas expertas. La tarea consistió en definir si los indicadores adaptados eran relevantes o muy relevantes como funcionalidad en el catálogo en línea. De esta forma, se logran tres criterios por indicador, con los cuales se obtiene un promedio de relevancia para cada indicador.

Posteriormente a la resolución de la tarea, se obtiene un promedio con base en los dos criterios fundamentales, para el establecimiento del porcentaje de relevancia de cada uno de los parámetros: la definición de los indicadores muy relevantes por parte de las personas expertas y la cantidad de indicadores por parámetro.

\section{Sexta fase. Validación final de la propuesta}

La validación final de la propuesta se realizó en los catálogos en línea del IIDH, el INAMU y la $\mathrm{CIDH}$. Este proceso contó con la colaboración de dos personas observadoras, las cuales tuvieron la responsabilidad de aplicar el instrumento en las instituciones mencionadas. La investigadora realizó la guía y coordinación de la aplicación del mismo.

El procedimiento consistió en realizar la aplicación del instrumento las veces que fueran 
necesarias hasta lograr un $100 \%$ de coincidencia en la evaluación efectuada por las observadoras. Es decir, los resultados de la validación debían ser concordantes en todos los aspectos valorados.

\section{RESULTADOS}

Con el propósito de lograr el más alto nivel de evidencias de validez de contenido en el instrumento propuesto en la investigación, se realizaron dos acciones concretas. Por un lado, la revisión del instrumento por un juicio de personas expertas y una prueba piloto de aplicación de la propuesta por parte de dos personas observadoras, especialistas en la disciplina.

La revisión del instrumento por parte de un juicio de personas expertas estuvo divida en dos tareas fundamentales. En primer lugar, debían determinar si cada parte del instrumento (parámetros, sub parámetros, indicadores y alcance) era representativa con la evaluación que se requería efectuar, o si era necesario agregar o eliminar algún punto. En segundo lugar, les correspondió realizar las modificaciones de redacción para facilitar la lectura y comprender mejor cada uno de los aspectos que integran el instrumento.

También, con el objetivo de ampliar las evidencias de validez, la investigación realizó una prueba piloto para la aplicación del instrumento en una unidad de información especializada.

La valoración se realizó con el apoyo de dos observadoras especialistas en bibliotecología, quienes tuvieron como tarea principal aplicar el instrumento en la biblioteca W. K. Kellogg, de la Universidad Earth. Esta prueba piloto se aplicó entre los días del 22 al 25 de junio del 2012. Esta unidad de información se especializa en temas de recursos naturales y agropecuarios, con énfasis en la región tropical húmeda del planeta; asimismo, cuenta con un amplio catálogo en línea.

Por otro lado y con el propósito de definir la relevancia de los parámetros, indicadores y sub parámetros, se consultó a tres personas pertenecientes al jurado de expertas. El resultado de esta consulta, en suma con la cantidad de indicadores por parámetro, produjo los siguientes datos:

\section{Parámetros}

Los porcentajes correspondientes a la relevancia de los parámetros de Búsqueda, Interfaz gráfica y Web Social fueron los siguientes: $46 \%, 41 \%$ y $13 \%$, respectivamente (Cuadro 1 ).

Cuadro 1. Porcentaje correspondiente a la relevancia del parámetro en el instrumento

\begin{tabular}{|l|c|}
\hline \multicolumn{1}{|c|}{ Parámetro } & Porcentaje \\
\hline Búsqueda & $46 \%$ \\
\hline Interfaz gráfica & $41 \%$ \\
\hline Web social & $13 \%$ \\
\hline
\end{tabular}

Fuente: Elaboración propia, 2012.

\section{Indicadores}

El Cuadro 2 muestra el porcentaje de indicadores muy relevantes en el sub parámetro de Búsqueda; es decir, de los 42 indicadores del parámetro, 29 fueron definidos como muy relevantes por las personas juezas de la investigación. Ese número equivale a un $69 \%$ de indicadores muy relevantes del total de estos.

Con respecto al parámetro de la Web social, también se presenta el Cuadro 3, que muestra el porcentaje de indicadores muy relevantes. De los 15 indicadores del parámetro, dos fueron definidos como muy relevantes; ese número equivale a un $13,3 \%$ de indicadores muy relevantes del total de estos.

El Cuadro 4 muestra el porcentaje de indicadores muy relevantes del parámetro Interfaz gráfica. Es decir, de los 31 indicadores del parámetro, 22 fueron definidos como muy relevantes 
Cuadro 2. Cantidad de indicadores en el parámetro de Búsqueda, y número y porcentaje de los indicadores muy relevantes

\begin{tabular}{|l|c|c|c|}
\hline \multicolumn{1}{|c|}{ Sub parámetro } & $\begin{array}{c}\text { Total de } \\
\text { indicadores }\end{array}$ & $\begin{array}{c}\mathbf{N}^{\circ} \text { de indicadores } \\
\text { muy relevantes }\end{array}$ & $\begin{array}{c}\% \text { de indicadores } \\
\text { muy relevantes }\end{array}$ \\
\hline 1. Página inicial & 8 & 5 & 62,5 \\
\hline 2. Llaves de entrada & 15 & 11 & 73,3 \\
\hline 3. Página de resultados & 14 & 9 & 64,3 \\
\hline 3. Gestión de contenidos & 5 & 4 & 80,0 \\
\hline Total & 42 & 29 & 69,0 \\
\hline
\end{tabular}

Fuente: Elaboración propia, 2012

Cuadro 3. Cantidad de indicadores en el parámetro Web social, y número y porcentaje de los indicadores muy relevantes

\begin{tabular}{|l|c|c|c|}
\hline Sub parámetro & $\begin{array}{c}\text { Total de } \\
\text { indicadores }\end{array}$ & $\begin{array}{c}\mathbf{N}^{\circ} \text { de indicadores } \\
\text { muy relevantes }\end{array}$ & $\begin{array}{c}\% \text { de indicadores } \\
\text { muy relevantes }\end{array}$ \\
\hline 1. Web social & 15 & 2 & 13,3 \\
\hline
\end{tabular}

Fuente: Elaboración propia, 2012

Cuadro 4. Cantidad de indicadores en el parámetro Interfaz gráfica, y número y porcentaje de los indicadores muy relevantes

\begin{tabular}{|l|c|c|c|}
\hline \multicolumn{1}{|c|}{ Sub parámetro } & $\begin{array}{c}\text { Total de } \\
\text { indicadores }\end{array}$ & $\begin{array}{c}\mathbf{N}^{\circ} \text { de indicadores } \\
\text { muy relevantes }\end{array}$ & $\begin{array}{c}\% \text { de indicadores } \\
\text { muy relevantes }\end{array}$ \\
\hline 1. Disposición visual & 10 & 9 & 90,0 \\
\hline 2. Ergonomía & 8 & 6 & 75,0 \\
\hline $\begin{array}{l}\text { 3. Sistema de ayuda a per- } \\
\text { sonas usuarias }\end{array}$ & 13 & 7 & 53,8 \\
\hline Total & 31 & 22 & 71,0 \\
\hline
\end{tabular}

Fuente: Elaboración propia, 2012 
por las personas juezas de la investigación; ese número equivale a un $71 \%$ de indicadores muy relevantes del total de estos.

La validación final de la propuesta se realizó en los catálogos en línea del IIDH, el INAMU y la CIDH. La aplicación del instrumento en los catálogos en línea de estas instituciones fue realizada por dos observadoras especialistas en bibliotecología.

De cada catálogo en línea aplicado en las instituciones se obtuvieron dos instrumentos debidamente llenos; posteriormente, se procedió a la revisión, análisis y depuración de ellos. En los indicadores donde hubo algún tipo de diferencia de criterio, se verificó el dato directamente con las observadoras, a fin de determinar si existía un problema de observación o de redacción del alcance.

La valoración del catálogo en línea continuó con los indicadores donde no existió coincidencia hasta obtener un $\mathbf{1 0 0 \%}$ de las mismas. Al final de la aplicación del instrumento, se obtuvo un único instrumento por catálogo en línea evaluado.

Esta validación permitió afinar el instrumento final que presenta la investigación.

\section{CONCLUSIONES}

El desarrollo y aplicación de esta propuesta metodológica ha permitido arribar a las siguientes conclusiones, las cuales se ordenan de acuerdo con los objetivos específicos:

\section{Caracterizar y analizar los métodos de evaluación aplicados a los catálogos en línea de acceso público en distintas unidades de información}

1. Tras analizar las distintas aportaciones al estudio de los catálogos en línea, se comprueba que todas ellas tienen en común el uso de un formulario de determinadas características deseables en la visualización de la información que facilita el catálogo en línea, además de la bibliográfica. Estas investigaciones corroboran la eficacia de esta metodología para el análisis de los catálogos en línea, y se comprueba la interoperabilidad de este tipo de instrumentos, es decir, es de fácil aplicación a cualquier tipo de unidad de información.

2. Desde finales de los años setenta y a lo largo de los años ochenta, cuando comenzó a difundirse el uso de los catálogos en línea de acceso público, se llevaron a cabo diferentes estudios con el objetivo de describir y comparar las características de los ya existentes en relación con las interfaces de las personas usuarias. Una de sus particularidades es que se diseñan con base en una o más metodologías de evaluación, es decir, en su diseño se utilizan los criterios definidos por varias autorías.

3. Las herramientas y servicios de la web social favorecen la comunicación interpersonal y han facilitado la gestión y difusión de información, ya para el 2011 se realizó un estudio de evaluación aplicando indicadores creados a partir de las características de esta nueva fase de internet.

\section{Adaptar un instrumento de evaluación de catálogos en línea de acceso público mediante una selección de los existentes e incorporando aspectos de la web social para uso de las unidades de información especializadas}

1. Se adaptó un instrumento de evaluación de catálogos en línea, derivado de estudios previos y de los principios de visualización de la información definidos por la IFLA, que permite a las unidades de información especializadas valorar las fortalezas y debilidades del 
catálogo en línea y preparar sus planes de mejora continua.

2. El proceso de elección de la metodología, que se quiere adaptar para la evaluación de catálogos en línea de acceso público, debe darse con base en un estudio y análisis pormenorizado del material bibliográfico existente sobre el tema. Esto permite adaptar el instrumento a las necesidades de las unidades de información y de las personas usuarias, de acuerdo con los avances tecnológicos y de comunicación.

3. Para el diseño y adaptación de un instrumento de evaluación, es indispensable definir una metodología que permita alcanzar el más alto nivel de validez de contenidos de los parámetros, indicadores y alcance, para lo cual es conveniente combinar técnicas como el criterio de personas expertas en el tema y la realización de una prueba piloto aplicada por observadoras, con el seguimiento y coordinación de la persona investigadora.

4. Es importante establecer un sistema de relevancia de los parámetros e indicadores dentro del instrumento, con el propósito de determinar su importancia como funcionalidad del catálogo en línea en entorno web.

\section{Validar la propuesta de evaluación aplicándola a los catálogos en línea del Instituto Nacional de las Mujeres, del Instituto Interamericano de Derechos Humanos y la Corte Interamericana de Derechos Humanos}

1. La validación del instrumento en tres unidades de información facilitó que se alcanzara un nivel más alto de validez de contenido, que permita a las instituciones seleccionadas llegar a conclusiones certeras para la mejora de sus respectivos catálogos en línea.
2. El nuevo reto para las unidades de información nacionales consiste en diseñar nuevos servicios que sean útiles para las personas usuarias y las invite a participar y aportar su conocimiento dentro de una filosofía cada vez más inclusiva y democrática.

\section{- Recomendaciones}

A partir de las conclusiones de esta investigación se presentan algunas de las recomendaciones:

\section{A las unidades de información}

El catálogo en línea en entorno web es un producto no acabado, que se mantiene en constante avance; por lo tanto, su evaluación debe constituir una tarea permanente en la unidad de información especializada, y los resultados deben brindar información oportuna para los objetivos de mejora. En esta tarea es indispensable el monitoreo de los avances en la materia de evaluación de los catálogos en línea, para tener al alcance las últimas propuestas de evaluación y medir la factibilidad de aplicación.

Asimismo, se deben realizar esfuerzos sostenidos por facilitar espacios de participación de las personas usuarias en la evaluación permanente de los catálogos en línea. Por tal razón, es importante desarrollar herramientas que inviten a la participación y profundización en las relaciones con las personas usuarias, lo cual se puede lograr colocando formularios de evaluación cortos en los catálogos, abriendo las posibilidades de interacción por medio de los chats (servicios de referencia en línea en tiempo real), correo electrónico y foros de discusión de grupos de personas usuarias afines.

En los procesos de evaluación continuos de las unidades de información especializadas, se hace indispensable la valoración periódica de la recuperación de la información; por sus indicadores básicos, esta debe ser efectiva, eficiente y usable para las personas usuarias. 
En este tipo de procesos de evaluación de los catálogos en línea en entorno web, se deben tomar en cuenta, además de la estructura de datos (el registro bibliográfico), las funcionalidades y su ergonomía.

Con el fin de mejorar el acceso de las personas a la información en los catálogos en línea, se considera fundamental la construcción de interfaces amigables y el desarrollo de estrategias para mejorar la accesibilidad y la usabilidad en la recuperación de datos bibliográficos.

Se recomienda, a las unidades de información especializadas, avanzar hacia la implementación de los servicios y herramientas de la web 2.0, como una filosofía que centra su atención principalmente en las personas usuarias. Esta actitud 2.0, se basa en el aporte que las personas usuarias brindan y en el aprovechamiento que otras puedan hacer de ellos, lo cual permite a las unidades de información crear un sistema de confianza y transparencia, pues la toma de decisiones es colectiva y no individual.

Entonces, es necesario poner al servicio de las personas usuarias las herramientas y aplicaciones de la web social, considerando que no es necesario esperar a desarrollar en forma completa el catálogo, sino que este se puede ir modificando paulatinamente. En dicha tarea, es fundamental el aporte de diferentes grupos participantes en la etapa de transformación de un catálogo en línea que aproveche las tecnologías de la web social.

\section{Para futuras investigaciones}

Desde el Doctorado en Bibliotecología, se recomienda explorar una línea de investigación acerca del impacto que los avances tecnológicos y de comunicación han tenido en Costa Rica, en las personas especialistas de la bibliotecología. Tal investigación indudablemente está relacionada con los planes de estudio de las universidades públicas y privadas que imparten la carrera, así como con el Colegio de Bibliotecarios de Costa Rica y sus planes de actualización continuos para las personas profesionales agremiadas.
Puede analizarse cuáles han sido los avances, obstáculos y limitaciones de los catálogos en línea en entorno web en las unidades de información en Costa Rica, y cuáles son sus proyecciones de desarrollo. Por su parte, el Doctorado podría establecer, de forma permanente, líneas de investigación en este tema que se encuentra siempre en constante cambio, con personas usuarias con demandas cada vez más específicas.

De igual manera, es conveniente realizar un directorio especializado sobre los catálogos en línea que existen en Costa Rica, las temáticas en la cuales se especializan, la población meta de dichos servicios de información y la institución a la cual pertenece, así como las políticas de intercambio de información, entre otros. Esto favorecería la búsqueda especializada de datos y se convertiría en un importante aporte de la academia.

\section{REFERENCIAS}

Fattahi, R. (1997). The relevance of cataloging principles to the online environment: an historical and analytical study. Tesis doctoral no publicada, University of South Wales, School for Information, Library and Archives Studies, Australia. Recuperado 10 de junio de 2012 de http://profsite.um.ac.ir/ fattahi/chaz.htm

Hildreth, C.R. (1987). Beyond Boolean: Designing the Next Generation of Online Catalogs. Library Trends, 35 (4) 647-667. Recuperado el 3 de abril de 2010 de http://www.ideals.illinois.edu/bitstream/handle/2142/750o/librarytrendsv35i4k_ opt.pdf?sequence $=1$

IFLA. (2005). Guidelines for online public access catalogue (OPAC) displays. The Hague: IFLA.

López Yepes, J. ed. (2004). Diccionario Enciclopédico de Ciencias de la Documentación. (1a. ed.) España: Editorial Síntesis.

Rodríguez Bravo, B. \& Alvite Díez, L. (2004). Propuesta metodológica de evaluación de interfaces de OPACs. INNOPAC versus UNICORN. Revista Especializada de Documentación Científica, 27 (1), 30-44. 
